\title{
COMPETENCIAS INVESTIGATIVAS CON BASE EN EL TRANSHUMANISMO. REVISIÓN SISTEMÁTICA
}

\author{
INVESTIGATIVE COMPETENCES BASED ON \\ TRANSHUMANISM. SYSTEMATIC REVIEW
}

\author{
Reina Cleopatra Mestanza Páez ${ }^{1}$ \\ Recibido: 2019-01-10 / Revisado: 2019-03-10 / Aceptado: 2019-04-01 / Publicado: 2019-07-01 \\ Forma sugerida de citar: Mestanza-Páez, R. C. (2019). Competencias investigativas con base en el \\ transhumanismo. Revisión sistémica. Retos de la Ciencia, 3(7), pp. 11-25. \\ https://doi.ora/10.53877/rc.3.7.20190701.02
}

\section{RESUMEN}

La pretendida transformación humana propiciada por el Transhumanismo, produciría individuos más aptos en cuanto a sus capacidades, siendo el objetivo del artículo, presentar algunas investigaciones sobre el transhumanismo, desde una percepción de cambio y mejora en los contextos universitarios para la construcción de un perfil investigador y potenciar el desempeño docente. Los textos que se analizaron, fue el resultado de la revisión sistemática narrativa de tipo cualitativo en la que se busca dar un panorama general del transhumanismo. Por lo que se puede concluir, que la relevancia de la formación del investigador en el ámbito académicodocente permite innovar conocimientos con base en los objetos de aprendizajes como una herramienta para la mejora pedagógica, aunado las competencias investigativas que se construyen en los actores del quehacer educativo al generar interés hacia la investigación.

Palabras clave: competencias investigativas, transformación humana, educación.

\section{ABSTRACT}

The intended human transformation for transhumanism, would produce individuals more suitable in their capacities, being the objective of the article, presenting a few investigations on transhumanism, from a perception of change and improvement in university contexts for the profile construction of an investigator and encourage teaching performance. The texts that have been analyzed, resulted in the qualitative narrative system revision that seeks to give a general panorama of transhumanism. In conclusion, the relevancy of the investigators formation in academic teaching field allows us to innovate knowledge based on learning objects

\footnotetext{
${ }^{1}$ Doctora en Psicología (Ph. D). Profesora en la Facultad de Psicología de la Universidad Central del Ecuador. Ecuador. E-mail: reinamestanza9@gmail.com
} 
as a tool for pedagogical improvement, together with the research skills that are built in the actors of the educational task by generating interest towards research.

Keywords: competitive investigations, human transformation, education.

\section{INTRODUCCIÓN}

Es inevitable pensar en la humanidad sin ser transformada por la tecnología que propone proyectar en un humano el aumento de su inteligencia por medios artificiales, modulando su propio estado psicológico. Siendo el biólogo Huxley (1927), quien introduce el término transhumanista, como la superación de la humanidad en virtud de la tecnología. Por su parte, Cardozo y Meneses (2014), sostienen que para los transhumanistas las nuevas tecnologías, tendrían mayor probabilidad al ampliar capacidades mentales y físicas propiciando el crecimiento personal más allá de las limitaciones biológicas. Además, se consideran que existen importantes motivaciones para sostener las capacidades humanas (Villarroel, 2015).

Por otro lado, Bostrom (2005), sostiene que es el transhumanismo es un movimiento intelectual y cultural que desea mejorar las capacidades humanas, que se expresan al incorporar conocimiento científico y tecnológico, para crear, modificar - validar. Aunado al uso de la tecnología para potenciar las competencias investigativas en los espacios universitarios, en cuanto a la mejora pedagógica (García, 2017), lo que a su vez incide en el adecuado uso de tecnologías que no vulnere la naturaleza humana para que no deje de ser precisamente humana. Por otra parte, se potencia la reflexión desde la visión del investigador transhumanista (Bostrom, 2011), sabiendo priorizar sus capacidades y competencias como base de la productividad, fundamental para el futuro de la actividad científica y alcanzar los fines en la educación superior. Para Quesada (2018), el transhumanismo tiene consciencia de que las tecnologías pueden ser peligrosas, a pesar de los beneficios, por eso, la ética juega un papel relevante para guiar la aplicación de la tecnología en el ser humano.

Los autores, coinciden en la presencia de la tecnología, como "mejora" en contextos de educación superior donde estos temas comienzan a discutirse. En este artículo, la tecnología se lo va a asociar con la palabra, "conocimiento". De modo que, la motivación por el trabajo interdisciplinario como parte de un proceso cultural en el campo de la investigación, propicia el equilibrio en las dimensiones del ser, e incide en la calidad de acompañamiento a los estudiantes durante el proceso de formación de la competencia investigativa para construir propuestas e innovaciones en la sociedad del conocimiento.

Si bien es cierto, por un lado, la universidad constituye el contexto propicio para encuentros e intercambio de saberes, aunado el accionar del docente al campo de la investigación, no obstante, es el momento para generar nuevos conocimientos en los estudiantes y por ende desarrollar competencias investigativas que posibiliten la producción científica en pro de la sociedad ecuatoriana.

Por otro lado, se puede decir que cuando existe producción de conocimiento durante el proceso de investigación, los estudiantes serán capaces de establecer relaciones teóricas y prácticas. Justo en ese momento, es cuando el rol del docenteinvestigador juega un papel esencial al preocuparse del significado que representa la práctica investigativa en el contexto educativo, lo epistemológico, los métodos y el compromiso con la universidad, para acompañar a los estudiantes en el proceso de investigación dirigidos a beneficiar a la sociedad en la que están inmersos. 


\section{METODOLOGÍA}

Se realizó una "revisión sistemática-narrativa de corte cualitativo para obtener una visión general de los estudios de investigación sobre "transhumanismo" (Aguilera, 2014). Este tipo de revisión se caracterizó por ser una selección de información de forma más o menos exhaustiva, sobre el tema. Búsquedas que se hicieron en bases de datos como Scielo, EBSCO, Springer y otras. El procedimiento consistió en ingresar a diferentes bases de datos únicamente en español y utilizar las palabras claves "Competencias investigativas", "transhumanismo", y se optó por elegir los encontrados de 1984 a 2018. Cabe señalar, que se tomó en cuenta, algunos capítulos de libros a pesar de ser de la década anterior, por contener información sobre el surgimiento del transhumanismo y los más recientes fueron seleccionados por estar relacionados con las competencias investigativas, tema central de la investigación. Los artículos y capítulos de libro encontrados, se depuraron y se recuperaron solo aquellos vinculados en Instituciones de Educación Superior. Por ser una revisión cualitativa no se presenta un análisis estadístico, es así como se presenta la evidencia en forma descriptiva.

\section{DESARROLLO}

\section{Evidencia empírica del desempeño docente}

Los estudios científicos sobre desempeño docente, su proceso en la adquisición de conocimientos para la optimización pedagógica y transferencia de los programas de desarrollo docente en la mejora de la calidad de los aprendizajes de los estudiantes universitarios (Feixas, Lagos, Fernández, Herriko y Sabaté ,2015), proponen modelos encaminados a evaluar el desempeño docente, cuyo resultado sostiene que la docencia es un proyecto institucional dirigido a elevar la calidad de la educación. Por su parte, Campos y Chinchilla (2009), realizaron una reflexión en torno a la importancia de replantear y resignificar la formación sistemática de competencias para la investigación, con el objetivo de asumir este proceso como un elemento clave y articulador en el desarrollo de los programas académicos de educación superior. Por otro lado, Díaz, Romero y Heredia (2012), investigaron sobre el empleo de las TIC en espacios universitarios, cuyo resultado permitió sostener que los estudiantes, adquieren competencias, toman conciencia de lo que han aprendido, desarrollan habilidades y reflexionan sobre su aprendizaje con base en la asesoría psicopedagógica y tecnológica.

Por otra parte, Parra, Tobón y López (2015), realizaron un estudio para fortalecer el conocimiento de la socioformación, de las estrategias didácticas y de evaluación en los docentes con el fin de potenciar el cambio de un modelo educativo centrado en la enseñanza a un modelo educativo focalizado en la formación integral acorde con los retos de la sociedad del conocimiento. La investigación se basó en las tutorías, cuyo resultado contribuye en la formación integral del estudiante. La investigación desarrollada por Rodríguez, Rabazo y Naranjo (2015), sobre la adquisición de la competencia de resolución de problemas, empleando como instrumento de medida el inventario Social Problem-Solving Inventory-Revised, evidenció que los estudiantes aumentan su adquisición de destrezas a lo largo de la carrera con el apoyo del docente.

El modelo pedagógico de formación en competencias genéricas, realizada por Montagud y Gandía (2015), ponen de manifiesto que, cuando se introducen cambios metodológicos en las guías docentes y se asume el modelo pedagógico, los 
resultados indican que la realización de un mayor número de actividades formativas incide en los resultados de aprendizaje de los estudiantes, Hernández, Tobón, y Guerrero (2016), realizan su investigación sobre los lineamientos de las políticas de evaluación docente, con el fin de reasignarle un verdadero sentido a través de una propuesta, la cual permita el desarrollo profesoral continuo. Los resultados contribuyen a la construcción de consensos para la toma de decisiones, que trasciendan en la consolidación de las comunidades educativas, el trabajo autónomo y la profesionalización docente. Por su parte, Cancino y Márquez (2015), analizan el sistema de evaluación de desempeño académico considerando, que es relevante y necesario para alcanzar mayor eficacia y eficiencia a nivel institucional.

En este sentido, se implementan procesos de investigación empírica para determinar el impacto de las rúbricas socioformativas en la evaluación del desempeño para trascender el enfoque de objetivos y contenidos, de tal manera, que la educación responda a los retos que plantea la sociedad del conocimiento. El resultado revela que las rúbricas permiten evaluar el desempeño en la realización de procesos y constituyen una oportunidad de mejoramiento en las prácticas educativas.

Por su parte, Flores, Gatica, Sánchez y Martínez (2016), investigaron la evolución y la transición de los distintos instrumentos asociados al proceso de evaluación del desempeño docente, concluyendo en que la evaluación del desempeño docente es uno de los pilares que pueden sustentar la calidad del sistema educativo. Jara y Díaz (2017), basan su investigación en identificar los lineamientos de las políticas de evaluación docente para reasignarle un verdadero sentido a través de una propuesta que permita el desarrollo profesoral continuo, exaltando el colegaje, el trabajo autónomo y la profesionalización docente.

Los sustentos teóricos presentados por los autores, permiten que la investigación sea considerada como una herramienta didáctica metodológica en contextos de educación superior, tema que está por debatirse. Con este antecedente, el docente en su proceso de autoconstrucción del conocimiento para desarrollar y potenciar las competencias investigativas en su formación profesional, debería asumir su rol investigador, para poner en práctica en el contexto educativo. Por lo tanto, el cambio se observará en medida que los estudiantes motivados por la investigación, desarrollen competencias durante su proceso de formación como investigador para construir conocimientos.

\section{Presencia del transhumanismo}

La tecnología vinculada a la investigación, evidencia el uso de nuevos métodos estadísticos para respaldar las investigaciones (Torro, 2016). Sin embargo, la humanidad busca ser mejores humanos, capaces de reconocer la importancia del rol en la sociedad vinculada con el desarrollo tecnológico (Warwick, 2016). Por tanto, la vinculación de la tecnología con la humanidad, es la oportunidad para usar los objetos de conocimiento en la formación de las capacidades investigativas del docente, para incidir en el aprendizaje de los estudiantes, potenciando el desarrollo de competencias (Damberger y Hebert, 2017).

Se reconoce la auto-transformación (More, 2010), los avances científicos y tecnológicos, en la búsqueda del mejoramiento humano (Diéguez, 2017), que permite trascender ciertos límites inherentes a la condición humana mediante uso de la tecnología para optimizar las competencias investigativas (Gutierro, 2017). A sabiendas, que el transhumanismo requiere de un análisis de reflexión profunda, y una puesta en práctica en el quehacer de la investigación, se presenta algunas 
definiciones (Tabla 1), para reflexionar sobre las pretensiones del transhumanismo en el siglo XXI.

Tabla 1.

Principales aportes en la definición Transhumanismo

\begin{tabular}{ll}
\hline Autor & Concepto \\
\hline More, M. (1992) & $\begin{array}{l}\text { "orden espontáneo" por la "sociedad abierta", promueve la descentralización } \\
\text { del poder y de la responsabilidad. }\end{array}$ \\
Pearce, D. (1992) & $\begin{array}{l}\text { Ética del utilitarismo hedonista, programa para eliminar el sufrimiento tanto } \\
\text { en los animales humanos como en el no-humano, por medio de la neuro- } \\
\text { tecnología avanzada. } \\
\text { ldea según la cual el ser humano debe mejorarse a sí mismo, a través de la } \\
\text { ciencia y la tecnología, ya sea desde el punto de vista genético o desde el } \\
\text { punto de vista ambiental y social. } \\
\text { Regulación de las nuevas tecnologías, para lograr así seguridad y garantizar } \\
\text { que los beneficios estarán disponibles para todos. } \\
\text { Bughes, J. (2004) }\end{array}$ \\
$\begin{array}{l}\text { Movimiento intelectual y cultural que afirma la posibilidad y la deseabilidad de } \\
\text { mejorar fundamentalmente la condición humana a través de la razón } \\
\text { aplicada, desarrollando y haciendo tecnologías } \\
\text { Búsqueda del mejoramiento humano, físico, mental, moral, emocional o de } \\
\text { otra índole, mediante procedimientos tecnológicos. } \\
\text { Trascender ciertos límites inherentes a la condición humana mediante uso de } \\
\text { la tecnología para optimizar las competencias investigativas. } \\
\text { Reflexión filosófica, ética y política para no reducir el ser humano a un ideal } \\
\text { de perfección materialista y mecanicista. }\end{array}$ \\
\hline Quesada, F. (2018)
\end{tabular}

Fuente: Se elaboró considerando las investigaciones de Vásquez y Solana (2015).

Lo anterior, da a pensar en ciertas dificultades que se podría enfrentar, durante el proceso de formación de competencias investigativas, en el deseo de contar con profesionales para trascender en el campo de la investigación, con base a generar motivación por una cultura investigativa en los estudiantes ávidos de conocimiento para enfrentar las oportunidades y amenazas de la ciencia. Por lo tanto, se requiere contar con profesionales comprometidos e identificados con las instituciones para dar todo su potencial al momento de plasmar sus competencias investigativas en beneficio de la comunidad educativa e incidir en el contexto.

\section{Necesidad de hablar sobre competencias investigativas}

En América Latina, la mayoría de las instituciones de educación superior se encuentran marginadas del proceso de la globalización educativa, haciendo uso de los productos elaborados por las comunidades científicas internacionales (UNESCO, 2013). Limitando la capacidad para interactuar creativamente con las redes investigativas y generar conocimientos relevantes para la ciencia y sus contextos.

Las competencias investigativas (Tabla 2), permiten reflexionar, sobre el ejercicio de la práctica pedagógica y adentrarse en el conocimiento, buscando la mejora de los procesos de enseñanza aprendizaje para el desarrollo de una cultura investigativa (Luque, Quintero y Villalobos, 2012) y mejorar la calidad de los productos en la práctica docente (García y Veleros, 2012). Por lo tanto, la investigación en el contexto universitario, fomenta la producción de conocimiento, la acreditación y aseguramiento de la calidad educativa (Sancho, Creus y Padilla, 2010).

Si bien, se puede observar que, a nivel internacional, la tendencia de la formación del profesorado en las universidades se ha ido decantando hacia enfoques holísticos y cada vez más complejos del desarrollo académico, similar a lo encontrado por Fernández y Márquez (2014), lo cierto es que todavía en muchas de 
ellas solo se organizan acciones formativas para el desarrollo docente. El acelerado desarrollo tecnológico y la incidencia en la educación es notoria, por tanto, se hace necesario usar la tecnología, mediante nuevos paradigmas pedagógicos, así también incorporar el uso de los objetos de aprendizaje como elementos de la planificación curricular, similar a las investigaciones de Ortuño, et. al., (2016).

En este sentido, crear espacios para promover experiencias significativas orientadas a la reflexión, autocrítica y solución de problemas relevantes en la comunidad educativa, así como el trabajo colaborativo entre las personas implicadas, no solo contribuirá al desarrollo de competencias investigativas interpersonales para transformar la realidad a partir de la solución de problemas del contexto, sino también, será una fundamental en el inicio de un proceso de alfabetización académica para mejorar la calidad de la educación.

\section{Tabla 2.}

Desarrollo de la competencia investigativa docente

Formación Docente

Relación armónicamente entre los sujetos implicados

Habilidad para generar y difundir conocimientos.

Agudiza la observación para que sus percepciones sean selectivas

Evalúa su propia acción, avances y limitaciones

Propone soluciones a los problemas

Detecta, demuestra y pone en acción

Jerarquiza, da sentido a los datos cualitativos y elabora categorías de significado

Selecciona y maneja técnicas de recolección de datos, como también la utilización de software

Comprende, conoce, analiza, compara y evalúa teorías, tendencias y metodologías vinculadas con procesos cognoscitivos e intelectuales.

\section{Competencia \\ Interpersonales}

Comunicativas

Observacionales

Reflexivas

Propositivas

Procedimentales

Analíticas

Tecnológicas

Cognitivas

Fuente: Se elaboró considerando planteamientos de Buendía, Zambrano e Insuasty (2017)

En relación a la formación investigativa Buendía, Zambrano e Insuasty (2017), sostienen que la fortaleza está en la aplicación de los conocimientos teóricos para potenciar los procesos de enseñanza y aprendizaje a partir del desarrollo de proyectos investigativos. Al respecto Campos y Chinchilla (2009), refieren que las competencias se deben promover en el futuro profesional para lograr autonomía y responsabilidad en diversos contextos, siendo parte de ellas las competencias para la investigación. La investigación en el ámbito de los modelos de desarrollo docente y el impacto de la formación, presentan un abanico de conocimiento empírico como lo sostienen Cancino y Márquez (2015), validado sobre cómo habría que diseñar la formación y en qué habría que poner la atención para descubrir la efectividad y el impacto de esa formación

Los objetos de aprendizaje constituyen una herramienta para desarrollar investigaciones, puesto que, el valor de la información como recurso de aprendizaje ha creado la necesidad de disponer de ella, compartirla y reutilizarla. Es así, como los Objetos de Aprendizaje cumplen con características que permitan su óptimo uso en el campo disciplinar para construir nuevos conocimientos (Ortuño, Morgado y Rodríguez, 2016).

En la práctica se espera que el docente posea la capacidad para orientar el aprendizaje, tenga conciencia crítica, busque acciones pertinentes para mejorar la realidad educativa, fomente grupos de pensamiento para compartir construcciones científicas y ponga en práctica los valores en su vida profesional. Así, como formar y 
desarrollar competencias en los futuros profesionales para desempeñar investigaciones que den respuesta a los requerimientos locales, nacionales e internacionales (Annas, Andrews e Isasi, 2002).

El aporte para los investigadores, es que los Objetos de Aprendizaje permitan, la articulación entre la investigación y la práctica pedagógica en el desarrollo de una cultura investigativa para tomar conciencia del rol que deben asumir como docentes autocríticos en el desarrollo de habilidades y destrezas en los estudiantes, fortaleciendo competencias para generar interés por la investigación. Sin embargo la falta de conocimiento sobre el manejo o el buen uso que se dé a esta herramienta por parte de docentes y estudiantes incidirá en el proceso de construcción de las competencias investigativas y por ende en la calidad de las investigaciones.

Por tanto, el desarrollo de la competencia investigativa, permite que los juicios se rijan por una racionalidad que ayuda a abandonar la actitud acientífica en relación con las interpretaciones prefijadas, proceso que, a su vez, da como resultado el cuestionamiento y la modificación de las creencias que suelen atar a los seres humanos. Por lo anterior, repensar sobre la formación de la investigación en la educación, implica no solo una ruptura con la tradicional forma de concebir la ciencia y la producción de conocimiento, sino también la adopción de una postura política frente a la función social del conocimiento, que permite tener claridad respecto al papel de la investigación en el desarrollo social. De esta manera permite profundizar desde la reflexión hermenéutica acerca de las implicancias de las categorías conceptuales que dan cuenta de la transdisciplinariedad en la investigación educativa.

\section{Incidencia de las competencias investigativas como práctica heurística}

La Universidad en una sociedad globalizada, es la que asigna al docente el rol como investigador, quien a su vez desarrolla en los alumnos competencias que lo puedan llevar a generar y a manejar conocimiento en su desempeño profesional, desde la posición de investigador experimentado al nuevo investigador quienes deben demostrar competencia investigativa. Entre ellas están las de observar situaciones problemáticas, proponer soluciones, comprender las implicaciones y perfeccionar la escritura para sistematizar los datos mediante el manejo de la tecnología (Reiban, Rodríguez y Zeballos, 2017). Competencias que son importantes considerar en las Instituciones de Educación Superior, para realizar investigaciones que den respuestas asertivas e innovativas a las exigencias de la sociedad. 
Gráfico 1.

Fases del método heurístico

Determinación de la propuesta de mejora, evaluación y encuadre de continuidad.

Aplicación de las estrategias planteadas
Identificación del problema social de la ciencia

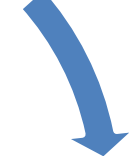

Definición y presentación del problema en cuestión

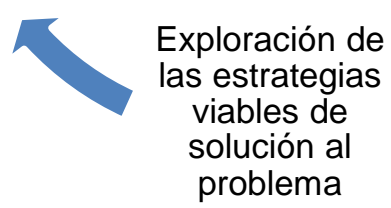

Fuente: Elaboración propia

En la práctica docente, se observa que los estudiantes universitarios presentan dificultades en la formación investigativa, por lo que surge la necesidad de potenciar habilidades que motiven al estudiante a incursionar el campo de la investigación (González y Flores, 2016). Por consiguiente, la universidad requiere replanteamientos y toma de decisiones para elevar su pertinencia social que implica revalorar la investigación en los espacios universitarios (Céspedes y Jiménez, 2011), constituyéndose clave en la gestión del conocimiento, la formación de recursos humanos altamente calificados y el trabajo de acción social. En ese sentido, surge la necesidad de potenciar líneas de investigación en el contexto universitario, dirigidas a la gestión del conocimiento y al desarrollo social a partir de una articulación entre la investigación, acción social y docencia, así como en la gestión curricular de la universidad para formar competencias investigativas. Por tanto, la competencia investigativa al momento de generar conocimiento prioriza la habilidad y la actitud frente a la visión como investigador transhumanista (Tutivén, 2018).

Los planteamientos de Bransford y Stein (1984), con base al método heurístico, sostienen que en un proceso de investigación es importante, identificar el problema social de la ciencia, definir y presentar el problema en cuestión, explorar las estrategias viables de solución al problema, aplicar estrategias planteadas, determinar la propuesta de mejora, evaluar y realizar un encuadre de continuidad. La presencia de la investigación propicia la solidez de la estructura universitaria, esencial en las competencias actitudinales, axiológica y medible del investigador académico. Siendo parte del investigador transhumanista, el reconocer la necesidad de potenciar la acción investigativa para desarrollar desde las disciplinas teorías o modelos en la comunidad académica e incidir en la formación del futuro profesional como gestor del conocimiento.

A la luz de lo anterior, las universidades requieren incorporar la investigación con mayor rigor en todos los niveles, no solo en los procesos de formación del estudiantado, sino también en el seno de las funciones académicas propias del quehacer universitario. Por ello, se debe promover la formación de competencias 
para la investigación como parte de la construcción de una cultura investigativa, reflexiva y orientada al desarrollo en los procesos académicos universitarios.

\section{El investigador transhumanista y su inclusión de la comunidad educativa}

Durante el último par de décadas, la academia ha comenzado a analizar las contribuciones del transhumanismo. Por su parte Bostrom (2005), sugiere desarrollar medios para explorar el "amplio espacio de los posibles modos de ser" que es inaccesible debido a limitaciones biológicas. So pesar que, la tecnología es el resultado de la aplicación de la ciencia, como actividades esencialmente humanas (Torrealba, Navas y Lara, 2018). Aunado, el potencial de mejoras en el bienestar humano con la transformación tecnológica, con base en la creatividad, que implica originalidad en esta época cambiante.

Entonces, en el caso específico de la formación en investigación, la incorporación de las tecnologías requiere, el diseño de un plan estratégico que permita migrar desde las modalidades que tradicionalmente se ha utilizado hacia la virtualidad. Para esto, es necesario definir con claridad los niveles y grados de incorporación de la virtualidad en las propuestas curriculares, al igual que todos los cambios de índole pedagógico y didáctico asociados a este proceso de conversión, incluyendo, además, la selección de las herramientas tecnológicas necesarias para fortalecer el desarrollo y la formación de las competencias en investigación que deberán desarrollar el personal académico y la población estudiantil.

Los científicos deben basar la reflexión con responsabilidad, dando prioridad a los intereses generales, fundamental para el futuro de la ciencia y la investigación. . De tal manera, el transhumanismo afirmaría que es deseable, mejorar la condición humana; aunque con ello, no refieren a mejoras conseguidas únicamente a través del sistema educacional (Garrido, Valdés y Arenas, 2007). Por su parte, la gestión académica cada vez cobra mayor importancia y está encaminada a hacer más eficiente los sistemas y procesos de gestión, ya que de esta forma se impulsa la investigación y en educación es necesario el logro de una mayor calidad y pertinencia de los procesos que lleva a cabo para enfrentar de mejor manera problemáticas del contexto (Segredo, 2015).

Los altos niveles de competitividad exigen nuevas formas de compromiso, de ver los hechos, de decidir y dirigir, de pensar y sentir, así como de gestionar las relaciones entre las instituciones de una forma más efectiva. Cabe señalar, que uno de los objetivos de las universidades es la difusión del conocimiento, siendo clave las personas que participan en ella, sin embargo, solo se podrá garantizar la calidad de la educación vinculando la docencia, con la investigación y la gestión universitaria para lograr la apropiación de líneas de investigación por los docentes en la construcción de propuestas innovadoras generando soluciones de índole, social, educativo, cultural y científico, potenciando las capacidades del ser humano en la praxis.

\section{RESULTADOS}

El análisis de la literatura revisada permite precisar que a las competencias investigativas se tratan desde diferentes perspectivas y enfoques. Sin embargo, se debería investigar sobre cuáles son las competencias investigativas que debe poseer el profesor universitario y como evaluar, ello se inscribe dentro de la problemática de la gestión por competencias en el ámbito educativo, sin ser necesariamente parte del reconocimiento del papel de la Universidad, en una 
sociedad globalizada en la que se debe reformular el papel del profesor como investigador (Reiban, Rodríguez y Zeballos, 2017). Consecuentemente, la competencia investigativa constituye una habilidad metodológica, que se desarrolla para la adquisición de saberes y solución de problemas en el campo de la investigación. Siendo que implica disponer de actitud e interés de conocer sobre los procesos de investigación, durante todo el proceso formativo para generar conocimiento. De esta manera, se puede enseñar y aprender a investigar con rigor metodológico en los espacios universitarios con base en la formación de competencias investigativas

El impacto del transhumanismo en los contextos universitarios, permitirá generar iniciativas y apropiación por parte de los investigadores, como mejora de la estrategia de investigación que incluye competencias pedagógicas, actitudinales y comunicativas para contribuir a la formación del investigador nobel, encaminado a potenciar habilidades de autonomía, competitividad, autoaprendizaje y adaptabilidad. En tales condiciones, el humanismo adquiere la contemporaneidad y la validez social más que nunca. Cómo trascender al humano en la educación, es por eso que los educadores necesitan interpretar la realidad educativa en términos de información, utilizando todas las ventajas que ofrecen los avances tecnológicos.

\section{DISCUSIÓN}

La evaluación docente y el desempeño docente son investigaciones realizadas por Tobón, Calderón, Hernández y Cardona (2015), Cancino y Márquez (2015), por su parte, Fernández, Sotolongo y Martínez (2016), basaron su investigación en conocer las percepciones de estudiantes y docentes, sobre un instrumento para evaluar el desempeño docente en cuatro dimensiones: competencias pedagógicasdidácticas, investigativas, de gestión académica y de extensión universitaria. Los resultados permitieron concluir sobre la efectividad de utilizar las cuatro dimensiones propuestas para evaluar el desempeño docente. De acuerdo con las percepciones de estudiantes y docentes, la competencia que priorizan en la evaluación del desempeño docente, es la competencia pedagógico-didáctica. Esto revela coincidencia con los resultados obtenidos en otras investigaciones precedentes realizadas por Cortés et al. (2014) donde se afirma que al estudiante principalmente le interesa lo que logra el docente con ellos y secundariamente otros aspectos.

Se reconocen las falencias en las competencias para escribir a nivel de la redacción de los informes investigativos e inconsistencias en su estructuración textual (Buendía-Arias, Zambrano-Castillo y Insuasty, 2018), siendo esencial del proceso investigativo la escritura académica, así como las actividades en pares para mejorar la producción. El uso del objeto de aprendizaje propuestos por Cárdenas, Guerra, y Soler (2017), será una fundamental, al incorporar competencias escriturales para generar documentos sólidos en su consistencia, estructura y contextualización producto de una investigación, cuyo propósito es el enriquecimiento del proceso de aprendizaje de los estudiantes, por ende, se contribuye al mejoramiento de la calidad de la educación

Se reconoce el papel de la educación superior, sin embargo, se hace necesario diseñar programas académicos que potencien habilidades para el desarrollo productivo de actividades de investigación e innovación con base en la apropiación y desarrollo de conocimientos para ejercer actividades vinculadas a la investigación científica, desarrollo tecnológico e innovación, como lo proponen Céspedes y Jiménez (2011). La formación de competencias para la investigación en el personal 
académico, se constituye en un proceso de construcción de aprendizaje así, quienes están implicados deben estar en constante actualización, desarrollar competencias para la investigación y gestionar estratégicas en docencia. Aunado las actividades de trabajo interdisciplinario y colaborativo entre docentes con experiencia en investigaciones y los que están iniciando su proceso de formación investigativa para contribuir a la producción de conocimientos.

Un reto en los programas académicos de educación superior, es la formación de competencias para la investigación, dado por la capacidad para identificar los problemas, elaborar propuestas de investigación que permitan dar solución, construir conocimientos y transformar esa realidad, coinciden con las propuestas de Feixas, et al., (2015). El potenciar las competencias en docentes como en los estudiantes que están incursionando líneas de investigación, posibilitará la construcción de proyectos innovadores, con apoyo de un investigador con mayor experiencia en contextos universitarios, con el objetivo de obtener propuestas innovadoras, similar a las investigaciones de Cortés, et al., (2014).

La apropiación pedagógica por parte del docente investigador, busca mejorar las competencias investigativas en los espacios universitarios, como parte integral del quehacer docente para interpretar, razonar y comunicar de forma eficiente conocimientos y fomentar en los estudiantes la construcción de las bases de su aprendizaje para incursionar en el campo de la investigación, así como lo proponen Reiban, et al., (2017). Sin embargo, para fortalecer los procesos de enseñanza y aprendizaje, es fundamental el desempeño del rol del docente como investigador para desarrollar la competencia investigativa en el entorno inmediato de acción, respondiendo a los retos que impone la modernización.

\section{CONCLUSIONES}

El advenimiento del transhumanismo en los contextos universitarios, posibilita el éxito de las Instituciones de Educación Superior, en cuanto a la calidad de sus producciones y a los proyectos que dan respuesta a las necesidades, sociales y culturales. Sin embargo, las competencias investigativas se deben construir en forma continua y sistemática para fortalecer el perfil investigador de docentes y estudiantes que están motivados por esta línea del conocimiento, dando cuenta del alcance pedagógico en los espacios universitarios.

En este mundo digital, la posibilidad de usar Objetos de Aprendizaje, abre oportunidades de comunicación en formas de enseñar y aprender. Por tanto, surge la necesidad de motivar procesos de investigación empírica desde la socioformación que contribuyan a consolidar la metodología de las rúbricas socioformativas como una respuesta a la formación de personas competentes, integrales y con un proyecto ético de vida sólido para afrontar los retos de la sociedad del conocimiento.

Se considera relevante en las competencias del investigador: la capacidad investigativa en cuanto a fundamentación teórica, practicidad de un proyecto, capacidad en difundir investigaciones, formar y dirigir recursos humanos en el campo de la investigación y formar grupos de investigación para generar publicaciones en acciones colaborativas intra e interinstitucionales, nacionales e internacionales.

Es así, como la investigación se incorpora en la academia, con base en la construcción y reconstrucción de acciones investigativas transformadoras en el desarrollo social desde una actitud científica. Siendo necesario, fortalecer en el estudiante la capacidad para: problematizar, gestionar conocimiento, valorar la 
relevancia de la investigación y sostener ideas fundamentadas. Por tanto, la sociedad del conocimiento, debería permitir reflexionar sobre la formación de competencias en la construcción de una cultura de investigación dirigida al desarrollo en los procesos académicos universitarios.

\section{REFENCIAS BIBLIOGRÁFICAS}

Aguilera, R. (2014). Revisión sistemática, revisión narrativa o meta análisis? Revista de la Sociedad Española del Dolor, 21(6), 359-360.Recuperado de http://scielo.isciii.es/scielo.php?script=sci_arttext\&pid=S1134-80462014000600010

Annas, G., Andrews, L. and R. Isasi, R. (2002). "Protecting the Endangered Human: Toward an International Treaty Prohibiting Cloning and Inheritable Alterations", American Journal of Law and Medicine 28 (2\&3):151-178. Recuperado de http://www.geneticsandsociety. org/downloads/2002_ajlm_annasetal.pdf

Bransford, J. y Stein, B. (1984). Solución IDEAL de problemas. Barcelona: Labor. Memory\&Cognition, $12 \quad$ (5), 522-529. Recuperado de https://link.springer.com/content/pdf/10.3758\%2FBF03198315.pdf

Bostrom, N. (2005). A History of trnashumanist Thought. Journal of Evolution and Technology. 14(1)1-25 Recuperado de http://jetpress.org/volume14/bostrom.html

Bostrom, N. (2011). Historia del pensamiento transhumanista. Argumentos de Razón Técnica, (14)157-191Recuperado de

http://institucional.us.es/revistas/argumentos/14/art_7.pdf

Buendía-Arias, X., Zambrano-Castillo, L. y Insuasty, E. (2018). El desarrollo de competencias investigativas de los docentes en formación en el contexto de la práctica pedagógica. Folios, 47, 179-195.

Cardozo, J. y Meneses, T. (2014). Transhumanismo: concepciones, alcances y tendencias. Análisis Revista Colombiana de Humanidades, 46(84),63-88 DOI: $10.15332 / \mathrm{s} 0120-8454$

Cárdenas, M., Guerra, C. y Soler, Y. (2017). Impacto social de la formación de competencias investigativas mediada por las TIC. Didáctica y Educación. 8(2) 117$136 \quad$ Recuperado http://www.runachayecuador.com/refcale/index.php/didascalia/article/view/1547

Campos, J. y Chinchilla, A. (2009). Reflexiones acerca de los desafíos en la formación de competencias para la investigación en educación superior. Revista Electrónica Actualidades Investigativas en Educación 9 (2) 1-20

Cancino, V. y Márquez, T. (2015). Evaluación de Desempeño de la Función Académica: Análisis de un Sistema en el Contexto Universitario Chileno. Formación Universitaria 8(3), 35-46

DOI: $10.4067 / S 0718-50062015000300005$

Céspedes, J. y Jiménez, A. (2011). Reflexiones acerca de los desafíos en la formación de competencias para la Investigación en Educación Superior. Actualidades Investigativas en Educación, 9(2) Recuperado de https://revistas.ucr.ac.cr/index.php/aie/article/view/9525

Cortés, E., Campos, M., y Moreno, P. (2014). Priorización de las dimensiones de evaluación del desempeño docente por el estudiante, en tres áreas del conocimiento, Formación universitaria, 7(2), 3-10

DOI: 10.4067/S0718-50062014000200002

Damberger, T y Hebert, E. (2017). Is Pedagogy Transhuman? Reflections on the Reltionship between Pedagogy and Transhumanism. Articles and Dissertations. 20(1). DOI: 10.12775/SPI.2017.1.002

Díaz-Barriga, F., Romero, E. y Heredia, A. (2012). Diseño tecnopedagógico de portafolios electrónicos de aprendizaje: una experiencia con estudiantes universitarios. Revista 
Electrónica de Investigación Educativa, 14(2), 103-1 Recuperado de http://redie.uabc.mx/vol14no2/contenido-diazbarrigaetal.html

Diéguez, A. (2017). Transhumanismo: La búsqueda tecnológica del mejoramiento humano. Barcelona: Herder. 248.

https://www.researchgate.net/profile/Antonio_Dieguez/publication/321490831_Trans humanismo_Propuestas_y_limites/links/5a254ff0aca2727dd87e98ab/Transhumanis mo-Propuestas-y-limites.pdf

Fernández, I., Eizagirre, A. y Madinabeitia, A. (2014). Constructing educational development from University Schools and Faculties: The case of the University of the Basque Country. Conference of International Consortium for Educational Development (ICED). Estocolmo. Recuperado de http:// www.iced2014.se/proceedings.html.

Fernandes, J. Sotolongo, M. y Martínez, C. (2016). La Evaluación del Desempeño por Competencias: Percepciones de Docentes y Estudiantes en la Educación Superior. Formación Universitaria 9(5), 15-24 DOI: 10.4067/S0718-50062016000500003

Feixas, M., Lagos, P., Fernández, I. y Sabaté, S. (2015). Modelos y tendencias en la investigación sobre efectividad, impacto y transferencia de la formación docente en educación superior. Educar, 51(1), 81-107 DOI: $10.5565 /$ rev/educar.695

Flores, F., Gatica, F., Sánchez, M. y Martínez, A. (2016). Evolución de la evaluación del desempeño docente en la Facultad de Medicina; evidencia de validez y confiabilidad. Investigación de educación médica. 6 (22) http://www.scielo.org.mx/scielo.php?pid=S200750572017000200006\&script=sci_artt ext\&tlng=pt

García, J. (2017). ¿Cómo mejorar al ser humano? Pedagogia e Vita 75 (2) 94-105. Recuperado https://www.researchgate.net/profile/Juan_GarciaGutierrez/publication/318653380_C OMO_MEJORAR_AL_SER_HUMANO_UN_ANALISIS_DE_LAS_TECNOLOGIAS_C ONVERGENTES_DESDE_LA_ANTROPOLOGIA_PEDAGŌGICĀ/links/5975a4f3a $\overline{6} f$ dcc83488e9280/COMO-MEJORAR-AL-SER-HUMANO-UN-ANALISIS-DE-LASTECNOLOGIAS-CONVERGENTES-DESDE-LA-ANTROPOLOGIAPEDAGOGICA.pdf

García, M. y Veleros, M. (2012). Competencias reflexivas y didácticas en profesores universitarios: Construcción de un portafolio electrónico.

Tendencias Pedagógicas, 20

Garrido, M., Valdés, L. M. y Arenas, L. (2007). El legado filosófico y científico del siglo XX. Madrid: Cátedra; cap. 35, pp. 867-886.

González, D. y Flores, V. (2016). Desarrollo de competencias investigativas en estudiantes de educación superior empleando técnicas lean ux. Caso: Delimitación de problemas de investigación: IUNAES. $10 \quad$ 131-143. http://iunaes.mx/inicio/wp-content/uploads/2016/12/REVISTA-VISI\%C3\%93NEDUCATIVA-IUNAES-22.pdf\#page $=139$

Gutierro, J. (2017). Transhumanismo. La búsqueda tecnológica del mejoramiento humano Oxímora.12, 248-252

DOI: 10.1344/oxi.2018.i12.20632

Hernández, J., Tobón, S. y Guerrero, G. (2016). Hacia una evaluación integral del desempeño: Las Rúbricas Socioformativas. Ra Ximhai, 12(6) ,359-376 Recuperado de: https://www.redalyc.org/articulo.oa?id=461/46148194025

Jara, N. y Díaz, M. (2017). Políticas de evaluación del desempeño del docente universitario, mito o realidad. Educación Médica Superior 31(2) http://www.medigraphic.com/pdfs/educacion/cem-2017/cem172r.pdf

Leyva, O. y Ganga, F. (2016). La formación por competencias en la educación superior: alcances y limitaciones desde referentes de México, España y Chile. México.Tirant humanidades.

Recuperado

de 
http://eprints.uanl.mx/10923/6/Libro_CU\%20GOBERNANZA\%20UNIVERSITARIA.pd $f$

Luque, D., Quintero, C. y Villalobos, F. (2012). Desarrollo de competencias investigativas básicas mediante el aprendizaje basado en proyectos como estrategia de enseñanza. Actualidades Pedagógicas, 60, 29-49

More, M. (2010). The Overhuman in the Transhuma. Journal of Evolution and Technology 21(1) 1-4. Recuperado de http://jetpress.org/more.htm

Montagud, M. y Gandía, J. (2015). Adquisición de competencias, actividades formativas y resultados del aprendizaje. ESTUDIOS SOBRE EDUCACIÓN. (28) 79-116 DOI: 10.15581/004.28.79-116

Ortuño, R., Morgado, E. y Rodríguez, C. (2016). Diseño de Objetos de Aprendizaje adaptados para cuatro estilos de aprender: un estudio de caso. Revista de Educação Pública, 25(59/2), 548-572. doi:http://dx.doi.org/10.29286/rep.v25i59/2.3834

Parra, H., Tobón, S. y López, J. (2015). Docencia Socioformativa y Desempeño Académico en la educación superior, Paradigma, 36, (1), 42 -55 https://www.researchgate.net/profile/Haydee_Parra2/publication/306346024_DOCEN CIA_SOCIOFORMATIVA_Y_DESEMPENO_ACADEMICO_EN_LA_EDUCACION_S UPERIOR/links/57b8f02208ae14f440bb51ce.pdf

Quesada, F. (2018). Transhumanismo": ¿Un nuevo humanismo? Un dilema fundamental para la bioética. Revista Estudios, (36). Recuperado de https://orcid.org/0000-00025164-4813

Rodríguez, E., Rabazo, A. y Naranjo, D. (2015). Evidencia empírica de la adquisición de la competencia de resolución de problemas. Perfiles Educativos | 37, (147), 50-64 https://reader.elsevier.com/reader/sd/pii/S0185269815000057?token=CAC32AE05F 911C90C440AAE82906B6E726AD886986C18863D10BDCFC3801B3B0639732870 87271A5C21EE11736281543

Reiban, R., Rodríguez, H. y Zeballos, J. (2017). Competencias investigativas en la Educación Superior. Publicando, 10 (1), 395-405 Recuperado de https://www.rmlconsultores.com/revista/index.php/crv/article/view/439/pdf_283

Sancho, J., Creus, A. y Padilla, P. (2010). Docencia, investigación y gestión en la Universidad: una profesión tres mundos. Praxis Educativa (Arg), XIV (14), 17-34. Recuperado de file:///C:/Users/Usuario/Documents/TRANSHUMANISMO/Sancho,CreusDOCENCIA, \%20INVESTIGACION\%20Y\%20GESTION.pdf

Segredo, A. (2016). Desarrollo organizacional. Una mirada desde el ámbito académico. Educación Médica. 17(1), 3-8. Recuperado de http://www.sciencedirect.com/science/article/pii/S1575181315000212

Torrealba, M., Navas, J. y Lara, N. (2018). Transhumanismo: Transdisciplinariedad en la investigación educativa. Revista Arbitrada del CIEG. 34(3) 93-100 Recuperado de http://www.grupocieg.org/archivos_revista/Ed.34\%20(93-100)-Torrealba\%20MaidaNavas\%20Jos\%C3\%A9\%20Antonio-Lara\%20Neomar_articulo_id417.pdf

Tutivén, C. (2018). Transhumanismo y subjetivación en la era de la técnica. (Tesis de maestría.) Universidad Casa Grande. Guayaquil. Recuperada de file:///C:/Users/Usuario/Documents/Transhumanismo/Tesis1810TUTt.pdf

Torro, L. (2016). Considering Human Vulnerability and Transhumanism: Some Theological Perspectives. Education and Humanities Research. 40 Recuperado https://doi.org/10.2991/icelaic-16.2017.166

UNESCO (2013). Situación educativa de América Latina y el Caribe: Hacia la educación de calidad para todos al 2015. Chile: UNESCO. Recuperado de https://www.google.com/search?client=firefox-bd\&q=UNESCO+Santiago\%2C+\%282013\%29\%2C+Situaci\%C3\%B3n+educativa+de +Am\%C3\%A9rica+Latina+y+el+Caribe\%3A+Hacia+la+educaci\%C3\%B3n+de+calida d+para+todos+al+2015.+Chile\%3A+UNESCO.

Vásquez, J. y Solana, E. (2015). Transhumanismo, neuroética y persona humana. 23 (3): 506- 13 http://dx.doi.org/10.1590/1983-80422015233087 
Villarroel, R. (2015). Consideraciones bioéticas y biopolíticas acerca del transhumanismo. El debate en torno a una posible experiencia posthumana. Revista de filosofía, 71, 177-190.

DOI: $10.4067 /$ S0718-43602015000100014

Warwick, K. (2016).Transhumanism: Some Practical Possibilities. FifF Kommunikation. (2): 24-27 Recuperado de http://www.fiff.de/publikationen/fiff-kommunikation/fk-2016/fk-20162/fk-2016-2content/fk-2-16-p24.pdf 